\title{
Lack of Association of BIRC5 Polymorphisms with Clearance of HBV Infection and HCC Occurrence in a Korean Population
}

\author{
Jin Sol Lee ${ }^{1}$, Jeong-Hyun Kim ${ }^{1}$, Byung Lae \\ Park $^{2}$, Hyun Sub Cheong ${ }^{2}$, Jason Y. Kim¹, \\ Tae Joon Park', Ji-Yong Chun', Joon Seol \\ $\mathrm{Bae}^{1}$, Hyo-Suk $\mathrm{Lee}^{3}$, Yoon Jun $\mathrm{Kim}^{3 *}$ and \\ Hyoung Doo Shin ${ }^{1,2 *}$
}

${ }^{1}$ Department of Life Science, Sogang University, Seoul 121-742, Korea, 'Department of Genetic Epidemiology, SNP Genetics, Inc., Rm 1407, Complex B, WooLim Lion's Valley, Seoul 153-801, Korea, ${ }^{3}$ Department of Internal Medicine and Liver Research Institute, Seoul National University, Seoul 110-744, Korea

\begin{abstract}
BIRC5 (Survivin) belongs to the inhibitor of apoptosis gene family. The BIRC5 protein inhibits caspases and consequently blocks apoptosis. Thus, BIRC5 contributes to the progression of cancer allowing for continued cell proliferation and survival. In this study, we identified eight sequence variants of BIRC5 through direct DNA sequencing. Among the eight single nucleotide polymorphisms (SNPs), six common variants with frequencies higher than 0.05 were selected for larger-scale genotyping $(n=1,066)$. Results of the study did not show any association between the promoter region polymorphisms and the clearance of hepatitis B virus (HBV) infection and hepatocellular carcinoma (HCC) occurrence. This is in line with a previous study in which polymorphisms in the promoter region does not influence the function of BIRC5. Initially, we were able to detect a signal with the $+9194 A>G$, which disappeared after multiple corrections but led to a change in amino acid. Similarly, we were also able to detect an association signal between two haplotypes (haplotype-2 and haplotype-5) on the onset age of HCC and/or HCC occurrence, but the signals also disappeared after multiple corrections. As a result, we concluded that there was no association between BIRC5 polymorphisms and the clearance HBV infection and/or HCC occurrence. However, our results might useful to future studies.
\end{abstract}

*Corresponding author: E-mail hdshin@sogang.ac.kr

Tel +82-2-705-8615, Fax +82-2-2026-4299

E-mail yoonjun@snu.ac.kr

Tel +82-2-2072-3081, Fax +82-2-743-6701

Accepted 1 December 2009
Keywords: BIRC5, survivin, hepatitis B virus (HBV), hepatocellular carcinoma (HCC), liver cirrhosis (LC), polymorphism

\section{Introduction}

Hepatitis B virus (HBV) infection, one of the most common virus infections among humans, is the major cause of acute and chronic liver diseases (Lin and Kao, 2008). It affects approximately 350 million people every year, especially those in Asia, Africa, Southern Europe, and Latin America (Lok and McMahon, 2007).

The clinical courses of HBV infection are diverse, ranging from spontaneous recovery after hepatitis to a chronic infection. The risk of developing liver cirrhosis (LC) or hepatocellular carcinoma (HCC) is higher for those who happen to be HBV chronic carriers as compared to the uninfected ones (Merican, Guan et al. 2000). Several previous studies on HBV infection have reported that polymorphisms were associated with the risk of HCC and/or clearance of HBV (Kida et al., 2007; Kim et al., 2006; Park et al., 2006; Shin et al., 2003).

BIRC5 (baculoviral inhibitor of apoptosis repeat-contaning 5), also known as survivin, is a protein encoded by the BIRC5 (Altieri 1994a; Altieri 1994b). It belongs to the inhibitor of apoptosis (IAP) gene family. The BIRC5 protein functions to inhibit caspase activation, thus leading to a decrease in apoptosis or programmed cell death. This has been shown by increase in apoptosis and decrease in tumor growth by disruption of BIRC5 induction pathways. In addition, the BIRC5 protein is highly expressed in most tumor cells (Sah et al., 2006). Because of this, BIRC5 is considered as one of the potent target for cancer therapy (Altieri, 2003).

A previous study demonstrates that BIRC5 is associated with microtubules of the mitotic spindle at the start of mitosis through the disruption of microtubule formation after BIRC5 protein in cancer cells are knocked out. This appearance leads to polyploidy as well as massive apoptosis (Castedo et al., 2004). Another study involves how the BIRC5 depleted cells exit mitosis without achieving proper chromosome alignment and then reforms single tetraploid nuclei. It suggests that BIRC5 protein is needed for sustaining mitotic arrest upon encounter with mitosis problems. The previous studies im- 
plicate that BIRC5 protein plays an important regulatory role both in the progression of mitosis and sustaining mitotic arrest (Castedo et al., 2004).

Based on those observations in cancer development, we hypothesized that BIRC5 polymorphisms may affect the function of BIRC5 protein and influence the clearance of HBV and HCC progression among HBV-infected patients thus, conducting a case-control study on the BIRC5 gene.

\section{Methods}

\section{Study population and outcomes}

A total of 1,066 Korean subjects having either present or past evidences of HBV infection were enrolled from the outpatient clinic of the liver unit or from the Center for Health Promotion of Seoul National University Hospital. Subjects were divided into two different groups according to serologic markers: the chronic carrier (CC) group, and the spontaneous recovery (SR) group. The CC and SR cohorts consisted of 632 and 434 subjects, respectively (Table 1). The diagnoses of the CC and SR subjects were established by repeated seropositivity for the hepatitis B surface antigen (HBsAg) (Enzygnost ${ }^{\mathbb{R}}$ HBsAg 5.0; Dade Behring, Marburg, Germany) over a 6-month period, and for both anti-HBs (Enzygnost ${ }^{\mathbb{R}}$ Anti-HBs II; Dade Behring, Marburg, Germany) and anti-HBc (AB-Corek; DiaSorin s.r.l., Saluggia, Italy) of the IgG type without HBsAg, respectively. Asymptomatic HBV carriers, which usually involve patients with inactive liver disease on liver biopsy, were also included the in CC group. However, it has been known that, HBV continues to replicate, albeit at very low levels, in some patients that have residual liver disease, which in most cases leads to the development of HCC. We excluded subjects who were positive for anti-HBs but not for an-
ti-HBc, as well as those positive for anti-HCV or anti-HIV (GENEDIA ${ }^{\circledR}$; Greencross Life Science Corp., Yongin-shi, Korea, $\mathrm{HCV}^{\mathbb{R}}$ 3.2; Dong-A Pharmaceutical Co., Seoul, Korea). Patients who had any other types of liver diseases such as autoimmune hepatitis, toxic hepatitis, primary biliary cirrhosis, and Budd-Chiari syndrome were also excluded from the study. None of the patients had any previous history of immunosuppression or anti-viral treatment. Informed consent was obtained from each patient, and the Institutional Review Board of Human Research at Seoul National University Hospital approved the study protocol. The clinical parameters are summarized in Table 1.

\section{Sequence analysis of the human BIRC5}

Using the ABI PRISM 3700 DNA analyzer (Applied Biosystems, Foster City, CA), we sequenced exons, introns, and the promoter region $(\sim 1.5)$ to discover variants from 24 unrelated individual' s DNA samples. Primer sets for the amplification and sequencing analysis of BIRC5 were designed based on GenBank sequences. Sequence analysis was carried out using Seq$\operatorname{Man}^{\circledR}$ software.

\section{Genotyping with fluorescence polarization detec- tion}

Amplifying primers and probes were designed for $\operatorname{TaqMan}^{\mathbb{R}}$ (An et al., 2002) which was used for genotyping of the six polymorphic sites. Primer Express (Applied Biosystems) was used to design both the PCR primers and the MGB TaqMan probes. One allelic probe was labeled with FAM dye and the other with fluorescent VIC dye. Typically, PCR was run in the TaqMan Universal Master mix without UNG (Applied Biosystems) at primer concentration of $900 \mathrm{nM}$ and TaqMan MGB-probe con-

Table 1. Clinical profile of the study subjects

\begin{tabular}{lccc}
\hline & & & $\mathrm{CC}$ \\
\cline { 3 - 4 } & $\mathrm{SR}$ & $\mathrm{CH}$ or LC & $\mathrm{HCC}$ \\
\hline No. of subjects & 434 & 325 & 307 \\
Age (mean (range)) & $55.1(22 \sim 79)$ & $49.4(22 \sim 81)$ & $59.2(24 \sim 79)$ \\
Sex (male/female) & $243 / 191$ & $265 / 60$ & $261 / 46$ \\
HBeAg (positive rate, \%) & 0 & 34.2 & 20.5 \\
HBeAb (positive rate, \%) & 0 & 30.8 & 45.9 \\
HBsAg (positive rate, \%) & 0 & 100 & 100 \\
HBsAb (positive rate, \%) & 100 & 0 & 0 \\
U albumin (positive rate, \%) & 0 & 5.8 & 13.0 \\
U blood (positive rate, \%) & 28.1 & 11.7 & 21.5 \\
\hline
\end{tabular}

SR: spontaneous recovery, CC: chronic carrier, $\mathrm{CH}$ : chronic hepatitis, LC: liver cirrhosis, HCC: hepatocellular carcinoma. 
centration of $200 \mathrm{nM}$. The reaction was performed in a 384-well format with a total reaction volume of 5 ul using $20 \mathrm{ng}$ of genomic DNA. The plate was then placed in a thermal cycler (PE 9,700, Applied Biosystems) and was heated for $2 \mathrm{~min}$ at $50^{\circ} \mathrm{C}$, then for $10 \mathrm{~min}$ at $95^{\circ} \mathrm{C}$, followed by 40 cycles of $95^{\circ} \mathrm{C}$ for $15 \mathrm{sec}$, and finally $60^{\circ} \mathrm{C}$ for $1 \mathrm{~min}$. The TaqMan assay plate was then transferred to a Prism 7900HT instrument (Applied Biosystems) where the fluorescence intensity of each well was read. Fluorescence data files from each plate were analyzed by automated software (SDS 2.1). Detailed information concerning the primers can be obtained at the website mentioned above.

\section{Statistics}

We examined Lewontin's D' (ID'l) and LD coefficient $r^{2}$ between all pairs of biallelic loci (Hedrick, 1987). Haplo- types of each individual were determined using the algorithm developed by Stephens et al.(Stephens et al., 2001), which (PHASE) uses a Bayesian approach incorporating priori expectations of haplotypic structure from population genetic and coalescent theory. Genetic effects of inferred haplotypes were analyzed in the same way as SNPs. Logistic regression models were used for calculating odds ratios ( $95 \%$ confidential interval) and corresponding P-values controlling for age (continuous value) and sex (male $=0$, female $=1$ ) as covariates. In our analysis on HCC occurrence, $\mathrm{LC}(\mathrm{LC}=1$, no $\mathrm{LC}=0)$ and $\mathrm{HBeAg}$ (negative=0, blank=1, positive=2) were also used as covariates. Statistical powers were also calculated using PGA matlab application (Menashe, Rosenberg et al. 2008). PGA is designed to calculate statistical power and other values of case-control genetic association studies. In this study, a co-dominant (1df) model, relative risk 1.3, disease prevalence value $7.1 \%$ (Lee, Kim

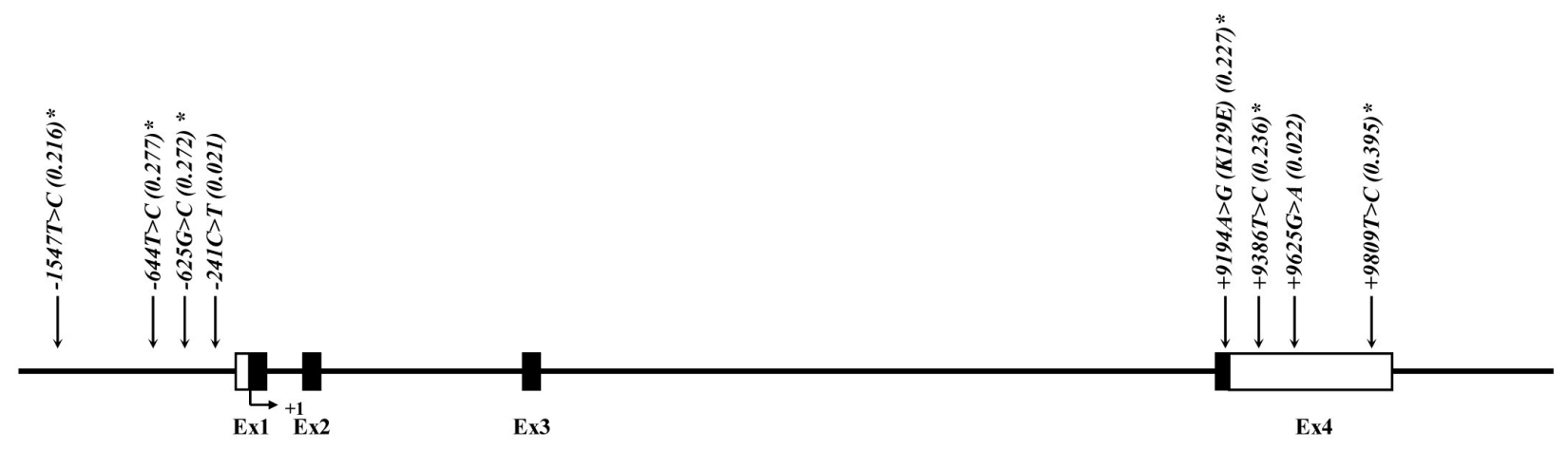

B. Haplotypes in BIRC5

C. LDs among BIRC5 polymorphisms

\begin{tabular}{|c|c|c|c|c|c|c|c|}
\hline Hap. & $\begin{array}{l}0 \\
\hat{N} \\
\frac{1}{2} \\
\frac{1}{2}\end{array}$ & \begin{tabular}{l}
0 \\
$\hat{f}$ \\
\multirow{J}{*}{} \\
$i$
\end{tabular} & 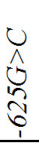 & $\begin{array}{l}0 \\
\hat{\Lambda} \\
\frac{\vec{t}}{2} \\
\frac{a}{a} \\
+\end{array}$ & 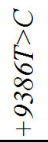 & $\begin{array}{l}u \\
\hat{\alpha} \\
\hat{\alpha} \\
\alpha \\
+ \\
+\end{array}$ & Freq. \\
\hline ht1 & $\mathrm{T}$ & $\mathrm{C}$ & $\mathrm{C}$ & $\mathrm{A}$ & $\mathrm{T}$ & $\mathrm{C}$ & 0.252 \\
\hline ht2 & $\mathrm{T}$ & $\mathrm{T}$ & G & G & $\mathrm{T}$ & $\mathrm{T}$ & 0.229 \\
\hline ht3 & C & $\mathrm{T}$ & G & A & $\mathrm{C}$ & $\mathrm{T}$ & 0.215 \\
\hline ht4 & $\mathrm{T}$ & $\mathrm{T}$ & G & A & $\mathrm{T}$ & $\mathrm{T}$ & 0.157 \\
\hline ht5 & $\mathrm{T}$ & $\mathrm{T}$ & G & A & $\mathrm{T}$ & C & 0.117 \\
\hline Others & & & & & & & 0.031 \\
\hline
\end{tabular}

\begin{tabular}{|c|c|c|c|c|c|c|c|}
\hline & & \multicolumn{6}{|c|}{$D^{\prime}$} \\
\hline & & 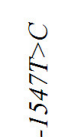 & \begin{tabular}{l}
$U$ \\
\multirow{J}{*}{} \\
\multirow{L}{*}{}
\end{tabular} & $\begin{array}{l}0 \\
\hat{\Lambda} \\
0 \\
\hat{0} \\
0\end{array}$ & $\begin{array}{l}0 \\
\frac{\Lambda}{d} \\
\frac{a}{a} \\
+\end{array}$ & 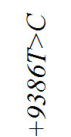 & $\begin{array}{l}0 \\
\hat{\alpha} \\
\hat{\sigma} \\
\stackrel{\alpha}{+} \\
+\end{array}$ \\
\hline \multirow{6}{*}{$r^{2}$} & $-1547 T>C$ & - & 1 & 0.984 & 1 & 0.991 & 0.983 \\
\hline & $-644 T>C$ & 0.108 & - & 0.993 & 1 & 0.73 & 0.987 \\
\hline & $-625 G>C$ & 0.101 & 0.969 & - & 1 & 0.71 & 0.99 \\
\hline & $+9194 A>G$ & 0.084 & 0.114 & 0.113 & - & 1 & 0.992 \\
\hline & $+9386 T>C$ & 0.885 & 0.063 & 0.058 & 0.093 & - & 0.819 \\
\hline & $+9809 T>C$ & 0.176 & 0.576 & 0.574 & 0.193 & 0.135 & - \\
\hline
\end{tabular}

Fig. 1. Gene maps and haplotypes of the BIRC5. (A) Polymorphisms identified in BIRC5. Coding exons are marked by shaded blocks and 5' and 3'UTR by white blocks. Asterisks $\left(^{*}\right)$ indicate SNPs that were genotyped in the larger population. The frequencies of SNPs without larger-scale genotyping were based on sequencing data. The first base of the translational start site is denoted as nucleotide +1 . (B) Haplotypes of BIRC5 in the Korean population. Only those with frequencies $\geq 0.05$ are shown. Others (1) contain rare haplotypes: TCACCC, TCATGC, TTATGC, TCGCGT, TCATGT, CTATGT, CTACCC, CTATGC, TCATCT, TTACGT and TTGTGC. C. LD coefficients (ID'l and $r^{2}$ ) among SNPs in BIRC5. 
et al. 1998), EDF (Effective degree of freedom) 2, and alpha error level $5 \%$ were used to calculate the statistical power.

\section{Results}

We identified eight genetic variants in BIRC5 using direct sequencing from 24 unrelated individuals. The range of direct sequencing included the promoter region, as well as the introns and exons of BIRC5. Among the eight genetic variants, four SNPs were located in the promoter region $(-1547 T>C,-644 T>C,-625 G>C$, $-241 C>T)$, one in exon $4(+9194 A>G)$ and three in 3' UTR $(+9386 T>C, \quad+9625 G>A, \quad+9809 T>C)$, respectively. The frequencies of each SNPs were 0.216 $(-1547 T>C), 0.277(-644 T>C), 0.272(-625 G>C)$, $0.021(-241 C>\pi), 0.227(+9194 A>G), 0.236(+93-$ $86 T>C), 0.022(+9625 G>A)$, and $0.395(+9809 T>C)$ (Fig. 1A, Table 2).

The logistic analysis for clearance of HBV infection, HCC occurrence, and onset age of HCC occurrence, as well as the p-values of each polymorphisms, haplotypes, and statistical powers are displayed in Table 3. In the case of SNPs, among the eight variants detected by direct sequencing, six were selected for the association analysis of the clearance of HBV infection and HCC occurrence. Two SNPs $(-241 C>T$ and $+9625 G>A)$ were excluded from the analysis due to low frequencies, 0.021 and 0.022 respectively. Among the six polymorphisms, $-644 T>C$ in the promoter region showed an association signal with HCC occurrence $(p=0.04)$ and onset age of HCC $(p=0.05)$ before correction was conducted, whereas the other SNPs in the promoter regions showed no association signals in all the analysis. An association with the clearance of HBV infection before correction was also detected in $+9194 A>G(p=0.04)$, which led to a change in amino acid lysine to glutamate.

Another association analysis was conducted for haplotypes. Five haplotypes with frequencies greater than $5 \%$ were selected for analysis. The selected haplotypes were able to explain more than $96 \%$ of the distribution (Fig 1B). Linkage disequilibrium coefficients (ID'l) between all SNP pairs were also shown (Fig. 1C). Among the haplotypes that we selected for analysis, haplotype-5 [T-T-G-A-T-C] showed an association with the clearance of HBV infection $(\mathrm{p}=0.01)$ and haplotype-2 [T-T-G-G-T-T] showed an association in onset age of HCC analysis $(p=0.03)$ before correction Table 3 .

\section{Discussion}

BIRC5 serves as a major factor in cancer development by contributing to the resistance of cancer cells to apoptosis (Hedrick, 1987). BIRC5 has been known to be involved in cell-cycle progression by bypassing cell-cycle checkpoints leading to continued proliferation (Ambrosini et al., 1998). This can be explained by the fact that tumor cells can divide without breaking and eventually survive. In addition, a previous study has reported that the expression level of BIRC5 in hepatocellular carcinoma cells is higher than in other cancer-causing cells (Montorsi et al., 2007). With the role played by BIRC5 in cancer development, we hypothesized that BIRC5 polymorphisms were associated with the clearance of HBV infection and/or HCC occurrence.

Previous studies have reported three polymorphisms $(-644 T>C,-625 G>C,-31 C>G)$ involved in the study of lung cancer in a Korean population $(n=582)$ (Jang et al., 2008) and seven polymorphisms (-267G>

Table 2. Frequency of BIRC5 gene polymorphisms in this study $(n=1,066)$

\begin{tabular}{|c|c|c|c|c|c|c|c|c|c|c|}
\hline \multirow{2}{*}{$\begin{array}{c}\text { Loci } \\
-1547 T>C\end{array}$} & \multirow{2}{*}{$\begin{array}{l}\text { Position } \\
\text { Promoter }\end{array}$} & \multirow{2}{*}{$\begin{array}{c}\text { rs\# } \\
r s 3764383\end{array}$} & \multirow{2}{*}{$\begin{array}{c}\text { Amino acid } \\
\text { change }\end{array}$} & \multicolumn{4}{|c|}{ Genotype } & \multirow{2}{*}{$\begin{array}{c}\text { Frequency } \\
0.216\end{array}$} & \multirow{2}{*}{$\begin{array}{c}\text { Heterozygosity } \\
0.339\end{array}$} & \multirow{2}{*}{$\begin{array}{r}\text { HWE } \\
0.107\end{array}$} \\
\hline & & & & $\mathrm{T}$ & $\mathrm{CT}$ & C & $\mathrm{N}$ & & & \\
\hline & & & & 677 & 351 & 60 & 1,088 & & & \\
\hline \multirow[t]{2}{*}{$-644 T>C$} & Promoter & rs8073903 & . & $\mathrm{T}$ & CT & C & $\mathrm{N}$ & 0.277 & 0.400 & 0.651 \\
\hline & & & & 556 & 434 & 79 & 1,069 & & & \\
\hline \multirow[t]{2}{*}{$-625 G>C$} & Promoter & rs8073069 & . & $\mathrm{G}$ & CG & C & $\mathrm{N}$ & 0.272 & 0.396 & 0.789 \\
\hline & & & & 561 & 415 & 80 & 1,056 & & & \\
\hline \multirow[t]{2}{*}{$+9194 A>G$} & Exon4 & rs17886532 & K129E & A & $A G$ & $\mathrm{G}$ & $\mathrm{N}$ & 0.227 & 0.351 & 0.195 \\
\hline & & & & 654 & 364 & 63 & 1,081 & & & \\
\hline \multirow[t]{2}{*}{$+9386 \mathrm{~T}>\mathrm{C}$} & Exon4 & rs2239680 & . & $\mathrm{T}$ & CT & C & $\mathrm{N}$ & 0.236 & 0.360 & 0.135 \\
\hline & & & & 640 & 372 & 69 & 1,081 & & & \\
\hline \multirow[t]{2}{*}{$+9809 T>C$} & Exon4 & rs1042489 & . & $\mathrm{T}$ & CT & $\mathrm{C}$ & $\mathrm{N}$ & 0.395 & 0.478 & 0.253 \\
\hline & & & & 401 & 494 & 176 & 1,071 & & & \\
\hline
\end{tabular}

p-values of deviation from Hardy-Weinberg Equilibrium (HWE). 


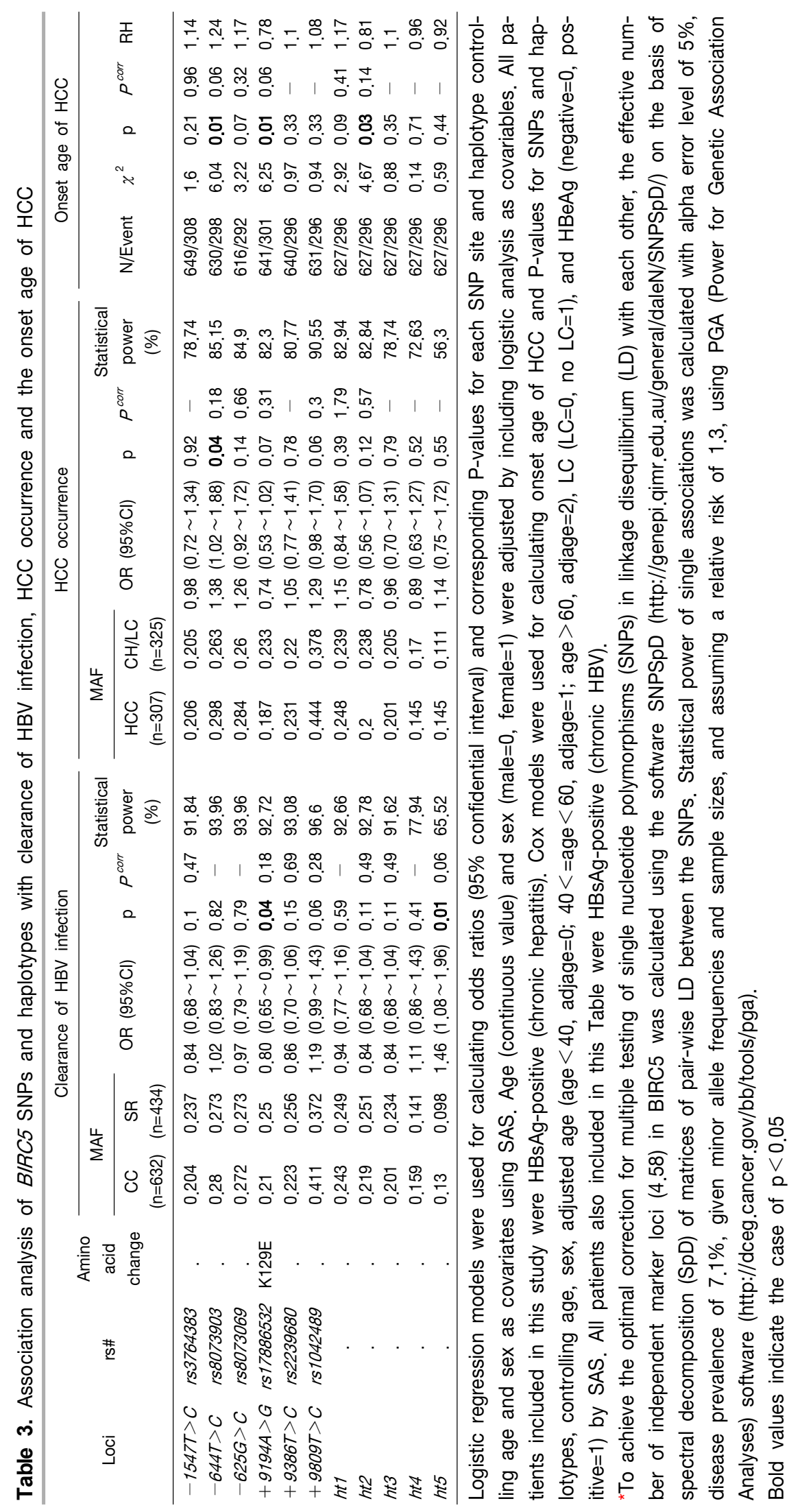


$A,-241 C>T,-235 G>A,-198,-191,-141$ and $-31 C>G$ ) studied for breast carcinoma in a French population ( $\mathrm{n}=191$ ) (Boidot et al., 2008). Among the promoter region polymorphisms that were studied in both Korean and French populations, $-31 C>G$ has been considered to play an important role in cancer development by affecting the expression level of mRNA of BIRC5 (Yang et al., 2009). Furthermore, two studies concerning cancer in Taiwan and China populations also examined the $-31 C>G$ polymorphism (Wang et al., 2009; Yang et al., 2009). In contrast, one study reported that the polymorphisms in the promoter region, including $-31 C<G$ does not have any influence on the BIRC5 activity (Boidot et al., 2008). Although $-31 C>G$ was not identified in our analysis, findings of this study on the promoter region polymorphisms $(-1547 T>C,-644 T>$ $C,-625 G>C$ ) showed no association signals with the clearance of HBV infection and/or HCC occurrence and is thus, consistent with one of the previous studies.

Among the six SNPs that were analyzed for largescale genotyping, $+9194 A>G$ initially showed an association signal with the clearance of HBV infection before multiple testing corrections. The polymorphism also demonstrated a protective effect on the clearance of HBV infection and led to the amino acid modification from lysine to glutamate. Marusawa et al. has demonstrated that BIRC5 forms complexes with a cellular protein called hepatitis B X-interacting protein (HBXIP). This protein's pro-caspase- 9 is activated by Apaf1, a cytoplasmic protein recognized for associating the $X$ protein of hepatitis B virus (HBX) and BIRC5. Complexes of BIRC5-HBXIP prevents the recruitment of components that can initiate apoptosis (Marusawa et al., 2003). Moreover, in another SNP study, Jang et al. reported that $+9194 A>G$ might be related with cancer (Jang et al., 2008). According to the study if alteration of amino acid by $+9194 A>G$ in exon4 affects protein formation, then it will also affect the interaction of HBXIP and BIRC5. In this study, $+9194 A>G$ initially showed an association signal with clearance of HBV infection. Although the signal disappeared after multiple testing corrections, it might provide valuable meaning to studies on the function of BIRC5.

In summary, we have identified eight polymorphisms in the human BIRC5 gene that were used to locate six common polymorphic sites selected for large-scale genotyping. After conducting a test on the functions of the promoter region polymorphisms, statistical analysis displayed no association among polymorphisms in the promoter region with the clearance of $\mathrm{HBV}$ infection and/or HCC occurrence. In addition, $+9194 A>G$ initially showed an association signal with the clearance of HBV infection and led to transformation of amino acid.
We also found that haplotype-2 and haplotype-5 initially showed association signals with HBV infection and the onset age of HCC analysis, respectively. However, all association signals disappeared after multiple testing corrections, which led us to conclude that there was no association between BIRC5 polymorphisms and the clearance of HBV infection and/or HCC occurrence. Although our $\mathrm{p}$-values did not undergo multiple testing corrections, results from this study might be useful for future researches which should include additional investigation on the function and/or expression of the polymorphisms of BIRC5.

\section{Acknowledgements}

We greatly acknowledge and thank the participants of the study and their families who took part in the HBV cohort study by Seoul National University. This work was supported by a grant from Korea Science and Engineering Foundation (KOSEF) funded by the Korea government (MEST) (No. 2009-0080157) as well as the National R\&D Program for Cancer Control, Ministry for Health, Welfare and Family affairs, Republic of Korea (0920280).

\section{References}

Altieri, D.C. (1994a). Molecular cloning of effector cell protease receptor-1, a novel cell surface receptor for the protease factor Xa. J. Biol. Chem. 269, 3139-3142.

Altieri, D.C. (1994b). Splicing of effector cell protease receptor-1 mRNA is modulated by an unusual retained intron. Biochemistry 33, 13848-13855.

Altieri, D.C. (2003). Validating survivin as a cancer therapeutic target. Nat, Rev. Cancer 3, 46-54.

Ambrosini, G., Adida, C., Sirugo, G., and Altieri, D.C. (1998). Induction of apoptosis and inhibition of cell proliferation by survivin gene targeting. J. Biol. Chem. 273, 11177-11182.

An, P., Nelson, G.W., Wang, L., Donfield, S., Goedert, J.J., Phair, J., Vlahov, D., Buchbinder, S., Farrar, W.L., Modi, W., O'Brien, S.J., and Winkler C.A. (2002). Modulating influence on HIV/AIDS by interacting RANTES gene variants. Proc. Natl. Acad. Sci. USA 99, 10002-10007.

Boidot, R., Vegran, F., Jacob, D., Chevrier, S., Gangneux, N., Taboureau, J., Oudin, C., Rainville, V., Mercier, L., and Lizard-Nacol, S. (2008). The expression of BIRC5 is correlated with loss of specific chromosomal regions in breast carcinomas. Genes Chromosomes Cancer 47, 299-308.

Castedo, M., Perfettini, J.L., Roumier, T., Andreau, K., Medema, R.H., and Kroemer, G. (2004). Cell death by mitotic catastrophe: a molecular definition. Oncogene 23, 2825-2837.

Hedrick, P.W. (1987). Gametic disequilibrium measures: proceed with caution. Genetics 117, 331-341. 
Jang, J.S., Kim, K.M., Kang, K.H., Choi, J.E., Lee, W.K., Kim, C.H., Kang, Y.M., Kam, S., Kim, I.S., Jun, J.E., Jung, T.H., and Park, J.Y. (2008). Polymorphisms in the survivin gene and the risk of lung cancer. Lung Cancer 60, 31-39.

Kida, A., Kakihana, K., Kotani, S., Kurosu, T., and Miura, O. (2007). Glycogen synthase kinase-3beta and p38 phosphorylate cyclin D2 on Thr280 to trigger its ubiquitin/proteasome-dependent degradation in hematopoietic cells. Oncogene 26, 6630-6640.

Kim, Y.J., Yoon, J.H., Kim, C.Y., Kim, L.H., Park, B.L., Shin, H.D., and Lee, H.S. (2006). IGF2 polymorphisms are associated with hepatitis $B$ virus clearance and hepatocellular carcinoma. Biochem. Biophys. Res. Commun. $346,38-44$.

Lee, M.S., Kim, D.H., Kim, H., Lee, H.S., Kim, C.Y., Park, T.S., Yoo, K.Y., Park, B.J., and Ahn Y.O. (1998). Hepatitis $B$ vaccination and reduced risk of primary liver cancer among male adults: a cohort study in Korea. Int. J. Epidemiol. 27, 316-319.

Lin, C.L., and Kao, J.H. (2008). Hepatitis B viral factors and clinical outcomes of chronic hepatitis B. J. Biomed. Sci. $15,137-145$.

Lok, A.S., and McMahon, B.J. (2007). Chronic hepatitis B. Hepatology 45, 507-539.

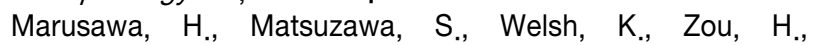
Armstrong, R., Tamm, I., and Reed, J.C. (2003). HBXIP functions as a cofactor of survivin in apoptosis suppression. Embo. J. 22, 2729-2740.

Menashe, I., Rosenberg, P.S., and Bingshu, E.C. (2008). PGA: power calculator for case-control genetic association analyses. BMC Genet. 9, 36.

Merican, I., Guan, R., Amarapuka, D., Alexander, M.J., Chutaputti, A., Chien, R.N., Hasnian, S.S., Leung, N.,
Lesmana, L., Phiet, P.H., Sjalfoellah Noer, H.M., Sollano, J., Sun, H.S., and Xu, D.Z. (2000). Chronic hepatitis B virus infection in Asian countries. J. Gastroenterol. Hepatol. 15, 1356-1361.

Montorsi, M., Maggioni, M., Falleni, M., Pellegrini, C., Donadon, M., Torzilli, G., Santambrogio, R., Spinelli, A., Coggi, G., and Bosari, S. (2007). Survivin gene expression in chronic liver disease and hepatocellular carcinoma. Hepatogastroenterology 54, 2040-2044.

Park, B.L., Kim, Y.J., Cheong, H.S., Kim L.H., Choi, Y.H., Lee, H.S., and Shin, H.D. (2006). Association of common promoter polymorphisms of MCP1 with hepatitis B virus clearance. Exp. Mol. Med. 38, 694-702.

Sah, N.K., Khan, Z., Khan, G., and Bisen, P. (2006). Structural, functional and therapeutic biology of survivin. Cancer Lett. 244, 164-171.

Shin, H.D., Park, B.L., Kim, L.H., Jung, J.H., Kim, J.Y., Yoon, J.H., Kim, Y,J., and Lee, H.S. (2003). Interleukin 10 haplotype associated with increased risk of hepatocellular carcinoma. Hum. Mol. Genet. 12, 901-906.

Stephens, M., Smith, N.J., and Donnelly, P. (2001). A new statistical method for haplotype reconstruction from population data. Am. J. Hum. Genet. 68, 978-989.

Wang, Y.H., Chiou, H.Y., Lin C.T., Hsieh, H.Y., Wu, C.C., Hsu, C.D., and Shen, C.H. (2009). Association between survivin gene promoter $-31 \mathrm{C} / \mathrm{G}$ polymorphism and urothelial carcinoma risk in Taiwanese population. Urology 73, 670-674.

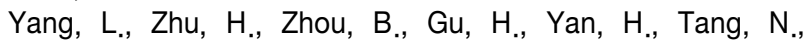

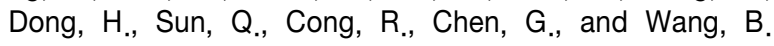
(2009). The association between the survivin C-31G polymorphism and gastric cancer risk in a Chinese population. Dig. Dis. Sci. 54, 1021-1028. 
202 Genomics \& Informatics Vol. 7(4) 195-202, December 2009

Table S1. Sequences of amplifying, Taqman probe, and extension primer for BIRC5 SNP genotyping

\begin{tabular}{|c|c|c|c|}
\hline Loci & rs\# & & Probe sequence \\
\hline \multirow[t]{4}{*}{$-1547 T>C$} & rs3764383 & Forward & GAGGAAGAAGCAGAGAGTGAATGTT \\
\hline & & Reverse & АCTCATATACCCTTCACCCAGATTTTCT \\
\hline & & VIC & TGTTTTGCCTATTTCCTT \\
\hline & & FAM & TTTTGCCTGTTTCCTT \\
\hline \multirow[t]{4}{*}{$-644 T>C$} & rs8073903 & Forward & CGATGTCTGCTGCACTCCAT \\
\hline & & Reverse & САССТСTGCAAAAAGCATCTACTC \\
\hline & & VIC & ATGAAGGACAAATGAACAG \\
\hline & & FAM & ATGAAGGACAAATGGACAG \\
\hline \multirow[t]{3}{*}{$-625 G>C$} & rs8073069 & Forward & AAAGACAGTGGAGGCACCAG \\
\hline & & Reverse & GCATGCCTGTAATCCCAACT \\
\hline & & Extension & ATGATTCCCCTGTTCATTTGTCCTTCATGCCC \\
\hline \multirow[t]{4}{*}{$+9194 A>G$} & rs17886532 & Forward & CAGGCAAAGGAAACCAACAATAAGA \\
\hline & & Reverse & GCAGCCAGCTGCTCGAT \\
\hline & & VIC & AACTGCGAAGAAAGT \\
\hline & & FAM & CTGCGGAGAAAGT \\
\hline \multirow[t]{4}{*}{$+9386 T>C$} & rs2239680 & Forward & GCAATGTCTTAGGAAAGGAGATCAACAT \\
\hline & & Reverse & CGCTGCACAGGCAGAAG \\
\hline & & VIC & AAGACAAAACAAGAGCACA \\
\hline & & FAM & AAGACAAAACAGGAGCACA \\
\hline \multirow[t]{3}{*}{$+9809 T>C$} & rs1042489 & Forward & GTTTTGATTCCCGGGCTTAC \\
\hline & & Reverse & AGCATCGAGCCAAGTCATTT \\
\hline & & Extension & TTGACTTGTGTGTGATGAGAGAATGGAGACAGAGTCCC \\
\hline
\end{tabular}

